\title{
Optimal maintenance of a production-inventory system with continuous repair times and idle periods
}

\author{
T. D. Dimitrakos* \\ Department of Mathematics \\ University of the Aegean \\ Karlovassi, 83200, Samos, Greece \\ E-mail: dimitheo@aegean.gr \\ *Corresponding author \\ C. C. Karamatsoukis \\ Hellenic Army Academy \\ Department of Mathematics and Engineering Sciences \\ Vari, 16673, Attica, Greece \\ E-mail: k.karamatsoukis@,fme.aegean.gr
}

\author{
E. G. Kyriakidis \\ Department of Statistics \\ Athens University of Economics and Business \\ 76 Patission Street, 10434, Athens, Greece \\ E-mail: ekyriak@aueb.gr
}

\begin{abstract}
In this paper two similar models for the maintenance of a production-inventory system are considered. In both models, an input generating installation supplies a buffer with a raw material and a production unit pulls the raw material from the buffer. The installation in the first model and the production unit in the second model deteriorate stochastically over time and the problem of their optimal preventive maintenance is considered. In the first model, it is assumed that the installation, after the completion of its maintenance, remains idle until the buffer is evacuated, while in the second model, it is assumed that the production unit, after the completion of its maintenance, remains idle until the buffer is filled up. The preventive and corrective repair times of the installation in the first model and the preventive and corrective repair times of the production unit in the second model are continuous random variables with known probability density functions. Under a suitable cost structure, semi-Markov decision processes are considered for both models in order to find a policy that minimizes the long-run expected average cost per unit time. A great number of numerical examples provide strong evidence that, for each fixed buffer content, the average-cost optimal policy is of control-limit type in both models, i.e. it prescribes a preventive maintenance of the installation in the first model and a preventive maintenance of the production unit in the second model if and only if their degree of deterioration is greater than or equal to a critical level. Using the usual regenerative argument, the average cost of the optimal control-limit policy is computed exactly in both models. Four numerical examples are also presented in which the preventive and corrective repair times follow the Exponential, the Weibull, the Gamma and the Log-Normal distribution, respectively.
\end{abstract}

Keywords-Maintenance; Production; Buffer; Semi-Markov decision processes; Control-limit policies; Expected average cost

\section{INTRODUCTION}

The maintenance of a production system is an important issue in modern industry. A failure of a system component may cause considerable delay in the production process, especially if the necessary time for its repair is long. The construction and analysis of a mathematical model for the description and optimal control of a deteriorating system component can result in substantial saving in operation and, also, in increased availability of the whole production system. In the last fifty years, a great number of maintenance problems have been studied and various mathematical models have been proposed for their solution. In Wang [19], a survey of different kinds of maintenance policies of deteriorating systems was given. The Markov decision model has been proved to be a powerful and flexible tool for the description and solution of many problems which are related to the optimal maintenance of a system. For example, in the papers of Federgruen and So [6], Douer and Yechiali [5], Van Der Duyn Schouten and Vanneste [18], Chen and Feldman [4], Benyamini and Yechiali [2], Moustafa, Maksoud and Sadek [12], Grosfeld-Nir [7], Tomasevicz and Asgarpoor [17], Kurt and Kharoufeh [10,11], Piunovskiy [14], Huang and Guo [8], Zhang and Gao [20], Bassey and Chigbu [1] and Borrero and Akhavan-Tabatabaei [3], suitable Markov decision models were constructed for various maintenance models. In the present paper we modify two models (see Karamatsoukis and Kyriakidis [9], Pavitsos and Kyriakidis [13]), in which the problem of the optimal maintenance of a production-inventory system was considered. Henceforth, we refer to Karamatsoukis and Kyriakidis [9] model as Model 1 and to Pavitsos and Kyriakidis [13] model as Model 2. In Model 1, a deteriorating installation supplies a buffer with a raw material and a production unit pulls the raw material from the buffer with constant rate. In Model 2, the raw material is transferred at a constant rate to a buffer and a deteriorating production unit pulls the raw material from the buffer. If the installation in Model 1 and the production unit in Model 2 are found to be at failed condition, a corrective maintenance must be commenced, while, if they are found to be at an operative condition, a preventive maintenance may be initiated. A suitable cost structure for the installation in Model 1 and for the production unit in Model 2 were introduced, including operating costs, maintenance costs, storage costs, shortage costs, penalty costs and costs due to the lost production. In both models, it was assumed that the maintenance times are geometrically distributed. Suitable discrete-time Markov decision processes were considered in both models in which 
stationary policies which minimize the long-run expected average cost per unit time were computed. It was proved that the average-cost optimal policy for each fixed buffer content is of control-limit type, i.e. it initiates a preventive maintenance of the installation in Model 1 and a preventive maintenance of the production unit in Model 2 if and only if the degree of their deterioration is greater than or equal to a critical level. In the present paper we modify the models by assuming that the preventive and the corrective repair times are continuous random variables which follow some known continuous distributions (e.g. Exponential, Weibull, Gamma, LogNormal). In Model 1, it is assumed that, after the completion of a preventive or a corrective maintenance, the installation remains idle until the buffer is evacuated. In Model 2, we assume that if the buffer is not full when a preventive or a corrective maintenance of the production unit is completed, the production unit remains idle until the buffer is filled up. When the repair times are continuous, it seems intuitively reasonable that the optimal policy is again of control-limit type in both models. However, rigorous proofs seem to be difficult in both models. Suitable semi-Markov decision processes are constructed and numerical results provide strong evidence that the average-cost optimal policies are again of control-limit type in both models. The average cost of a control-limit policy is computed exactly in both models by applying the usual regenerative argument. The rest of the paper is organized as follows. The description of Model 1 is given in the next section and the description of Model 2 is given in Section III. In Section IV, we briefly describe how the average cost of a control-limit policy can be computed exactly in both models. In Section V, two numerical examples for Model 1 and two numerical examples for Model 2 are presented in which the preventive and corrective repair times follow the Exponential, the Weibull, the Gamma and the Log-Normal distribution, respectively.

\section{MODEL 1}

We consider a production-inventory system which consists of an installation (I) that supplies a buffer (B) with a raw material and a production unit $(\mathrm{P})$ which pulls the raw material from the buffer with a constant rate equal to $d$ (units/time). The buffer has finite capacity which is equal to $K$ units of raw material and has been built between the production unit and its input generating installation to cope with unexpected failures of the installation. As long as the buffer capacity is not reached, the installation operates at a constant rate of $p$ units of raw material per unit time $(p>d)$ and the excess output is stored in the buffer. As long as the buffer is filled up, the installation reduces its speed from $p$ to $d$. The three components of the system are depicted in Figure 1.

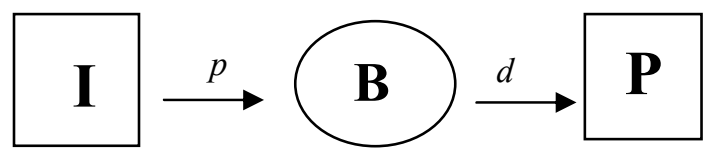

Fig. 1. The three components of the system
As mentioned in Van Der Duyn Schouten and Vanneste [18], an example of this production system could be an offshore oil exploration platform which provides the crude oil to onshore refineries. The crude oil is transported by pipelines from the platform to storage tanks from which is further transported to the refineries. In this case, the crude oil, the exploration platform, the refineries and the storage tanks are the raw material, the installation, the production unit and the buffer, respectively. We suppose that the installation deteriorates as time evolves and it is monitored at discrete, equidistant time epochs $\tau=0,1, \ldots$ (say every day) and a decision must be made at each epoch. There are three possible actions $a \in\{0,1,2\}$ which are selected at each time epoch. The possible actions are: (i) the action of allowing the installation to operate $(a=0)$ (ii) the action of starting a preventive maintenance of the installation $(a=1)$ and (iii) the action of starting a corrective maintenance of the installation $(a=2)$. A policy is any rule for choosing actions at each decision epoch. A policy is said to be stationary, if at a time epoch it chooses one action which depends only on the current state of the process. The state of installation at each decision epoch is classified into one of $m+2$ working conditions $0,1, \ldots, m+1$, which describe increasing degrees of deterioration. State 0 represents a new installation before any deterioration occurs while state $m+1$ represents the failure state of the installation. The intermediate states $1, \ldots, m$ are operative. If at a time epoch $\tau$ the state of the installation is operative and the content of the buffer is $x<K$, then the content of the buffer at the next time epoch $\tau+1$ will be $\min (x+p-d, K)$. This increase of the buffer content will happen even if the state of the installation at the time epoch $\tau+1$ is the failure state $m+1$. The transition probability of moving from working condition $i$ at time epoch $\tau$ to working condition $j$ at time epoch $\tau+1$ is equal to $p_{i j}$. We assume that the probability of eventually reaching the condition $m+1$ from any initial state $i$ is nonzero. If at a time epoch the installation is found to be at the working condition $m+1$, then the action $a=2$ is compulsory. If at a time epoch the installation is found to be at state $i \in\{1, \ldots, m\}$, then we may choose either action $a=0$ or action $a=1$. Both preventive and corrective maintenance are nonpreemptive, i.e. they cannot be interrupted and they bring the installation to the perfect working condition 0 . It is assumed that the preventive and the corrective repair times are continuous random variables with known probability density functions $f_{1}(x)$ and $f_{2}(x)$, respectively. The cost rates during a preventive and a corrective maintenance are equal to $c_{p}$ and to $c_{f}$, respectively. If at a time epoch the installation is found to be at state $i \in\{0, \ldots, m\}$ and the action $a=0$ is chosen, an operating cost is incurred until the next time epoch, which is equal to $c_{i}$, if the buffer is not full, or to $\widetilde{c}_{i}$, if the buffer is full. We suppose that, during any maintenance (preventive or corrective) of the installation, the buffer is not supplied with raw material. If during a preventive or a corrective maintenance the buffer is empty, then the operation of the 
production unit stops. A shortage cost is incurred when a preventive or a corrective maintenance is performed and the buffer is empty. The unit of cost has been chosen in such a way so that, the shortage cost rate is equal to the lost demand $d$ for each unit of time during which a preventive or a corrective maintenance is performed. We also suppose that the cost of holding a unit of the raw material in the buffer for one unit of time is equal to $h>0$. We assume that, if the buffer is not empty when a preventive or a corrective maintenance is completed, the installation does not resume its operation immediately but it remains idle until the buffer is evacuated. As soon as the buffer is evacuated, the installation restarts its operation by supplying the buffer with raw material at a rate equal to $p$ (units/time). Let $m_{P M}$ and $m_{C M}$ be the expected times required for a preventive and a corrective maintenance, respectively. The following conditions on the cost structure and on the transition probabilities are assumed to be valid:

Condition 1. $c_{0} \leq c_{1} \leq \ldots \leq c_{m}, \quad \widetilde{c}_{0} \leq \widetilde{c}_{1} \leq \ldots \leq \widetilde{c}_{m}$. That is, as the working condition of the installation deteriorates, the operating cost increases.

Condition 2. $\tilde{c}_{i} \leq c_{i}, 0 \leq i \leq m$. That is, the reduction of the speed from $p$ (units/time) to $d$ (units/time) of the installation, as soon as the buffer is filled up, causes a reduction of its operating cost.

Condition 3. $m_{P M} \leq m_{C M}$. That is, the expected time required for a preventive maintenance of the installation is smaller than the expected time required for a corrective maintenance.

Condition 4. $c_{p} \leq c_{f}$. That is, the cost rate of a preventive maintenance does not exceed the cost rate of a corrective maintenance.

Condition 5. (An Increasing Failure Rate Assumption). For each $k=0, \ldots, m+1$, the function $D_{k}(i)=\sum_{j=k}^{m+1} p_{i j}$ is nondecreasing in $i, 0 \leq i \leq m$.

For the present problem, the state space of the system is the set $S=\{0,1, \ldots, m+1\} \times\{0, \ldots, K\}$, where $(i, x) \in S$ is the state in which $i$ is the working condition of the installation and $x$ is the content of the buffer. We consider a semi-Markov decision process in which we aim to find a policy that minimizes the long-run expected average cost per unit time. The relevant theory of semi-Markov decision processes can be found in Section 3.5 in Tijms's [16] book. The decision epochs in our problem are the time epochs at which the system enters a state in $S$. Let $p_{s l}(a), a \in\{0,1,2\}$, be the probability that the state of the system at the next decision epoch will be the state $l \in S$, if the present state is $s \in S$ and the action $a \in\{0,1,2\}$ is selected and let $T(s, a)$ and $C(s, a)$ be the corresponding expected transition time and cost, respectively. These quantities are given below.

$$
\begin{aligned}
& p_{(i, x)(j, \min (x+p-d, K))}(0)=p_{i j}, 0 \leq i \leq m, 0 \leq j \leq m+1,0 \leq x \leq K, \\
& T((i, x), 0)=1,0 \leq i \leq m, 0 \leq x \leq K, \\
& C((i, x), 0)=c_{i}+h x, 0 \leq i \leq m, 0 \leq x<K, \\
& C((i, K), 0)=\widetilde{c}_{i}+h K, 0 \leq i \leq m, \\
& p_{(i, x)(0,0)}(1)=1,0 \leq i \leq m, 0 \leq x \leq K,
\end{aligned}
$$$$
T((i, x), 1)=m_{P M}+\int_{0}^{x / d}\left(\frac{x}{d}-t\right) f_{1}(t) d t, 0 \leq i \leq m, 0 \leq x \leq K,
$$$$
C((i, x), 1)=c_{p} m_{P M}+\frac{h x^{2}}{2 d}+\int_{x / d}^{\infty}(t d-x) f_{1}(t) d t,
$$$$
0 \leq i \leq m, 0 \leq x \leq K,
$$$$
p_{(m+1, x)(0,0)}(2)=1,0 \leq x \leq K,
$$$$
T((m+1, x), 2)=m_{C M}+\int_{0}^{x / d}\left(\frac{x}{d}-t\right) f_{2}(t) d t, 0 \leq x \leq K,
$$$$
C((m+1, x), 2)=c_{f} m_{C M}+\frac{h x^{2}}{2 d}+\int_{x / d}^{\infty}(t d-x) f_{2}(t) d t,
$$

$0 \leq x \leq K$

We will explain in detail how (1) is derived. Suppose that the state of the system at a decision epoch is the state $(i, x), 0 \leq i \leq m, 0 \leq x \leq K$. If the action $a=1$ of preventive maintenance is initiated, then the state of the system at the next decision epoch will be the state $(0,0)$. The corresponding onestep expected cost $C((i, x), 1)$ consists of the expected maintenance cost, the expected holding cost and the expected shortage cost. The expected maintenance cost is equal to $c_{p} m_{P M}$, the expected holding cost is equal to $h \int_{0}^{x / d}(x-t d) d t$ and the expected shortage cost is equal to $\int_{x / d}^{\infty}(t d-x) f_{1}(t) d t$. By summing the above three expressions, we obtain (1). A great number of numerical examples, in which the optimal policies were found by implementing the standard value-iteration algorithm for semi-Markov decision processes (see pp. 222223 in Tijms [16]), provide strong evidence that, for each fixed buffer content $x \in\{0, \ldots, K\}$, the optimal policy is of controllimit type. It seems difficult to prove this conjecture.

\section{MODEL 2}

We consider a production system in which the raw material is transferred into a buffer (B) at a constant rate $p$ (units/time) and a production unit $(\mathrm{P})$ pulls the raw material from the buffer. The capacity of the buffer is fixed and it is assumed to be equal to $K$ units of raw material. The production unit is 
subjected to deterioration. As long as the buffer is not empty and the production unit is in operative condition, the production unit pulls the raw material at a constant rate of $d$ (units/time). We assume that $d>p$. When the buffer is empty and the production unit is in operative condition, the production unit reduces its pull-rate from $d$ to $p$. As mentioned in Pavitsos and Kyriakidis [13], an example of this system could be the case in which the production unit is a power station. The raw material, that is oil or gas, is transferred at a constant rate to a storage tank (buffer) and the power station pulls it and converts it to electricity. The production unit deteriorates stochastically over time. We assume that it is monitored at discrete, equidistant time epochs $\tau=0,1, \ldots$ (say every day) and is classified into one of $m+2$ working conditions $0,1, \ldots, m+1$, which describe increasing levels of deterioration. Working condition 0 means that the production unit is new or functioning as good as new, while condition $m+1$ means that the production unit is in failed (inoperative) condition and it cannot pull the raw material from the buffer. The intermediate states $1, \ldots, m$ are operative. If at a time epoch $\tau$ the working condition of the production unit is $i<m+1$ and the buffer contains $x>0$ units of raw material, then the content of the buffer at time epoch $\tau+1$ will be $\max (x+p-d, 0)$. This decrease of the buffer content will happen even if the working condition of the production unit at time epoch $\tau+1$ is $m+1$. If at a time epoch $\tau$ the working condition of the production unit is $i<m+1$ and the buffer is empty, then the buffer will remain empty at time epoch $\tau+1$, since in this case the production unit pulls the raw material from the buffer with rate $p$ (units/time). It is assumed that if at a time epoch $\tau$ the working condition of the production unit is $i$ and the buffer content is $x$ then at time epoch $\tau+1$ the working condition of the production unit will be $j$ with probability $p_{i j}$. We suppose that the probability of eventually reaching the working condition $m+1$ from any initial state $i$ is nonzero. If the production unit at a time epoch is found to be at any working condition $i, i \leq m$, a preventive maintenance may be started. If the production unit at a time epoch is found to be at the working condition $m+1$, then a corrective maintenance must be commenced immediately. Both preventive and corrective maintenance are nonpreemptive, i.e. they cannot be interrupted and they bring the production unit to the working condition 0 . The preventive and the corrective repair times are random and we assume that they are continuous random variables with known probability density functions $f_{1}(x)$ and $f_{2}(x)$, respectively. When a preventive or a corrective maintenance of the production unit is performed, the buffer is supplied with the raw material at a constant rate of $p$ (units/time) until it is filled up. As long as the preventive or the corrective maintenance of the production unit lasts, the production process is interrupted. We suppose that the cost due to the lost production during a unit of time in which a preventive or a corrective maintenance of the production unit is performed is equal to $C>0$. When the buffer is not empty and the production unit pulls the raw material from the buffer with rate $d$, we do not incur a cost due to the lost production. However, when the buffer is empty, the production unit pulls the raw material from the buffer with rate $p$ which is smaller than $d$. It is reasonable to assume that in this case the cost due to the lost production during a unit of time is equal to $[(d-p) C] / d$. A penalty cost which is equal to $P>0$ is also imposed for each unit of raw material that it is not stored in the buffer during a preventive or a corrective maintenance of the production unit when the buffer is full. This cost is due to the necessary labor for transferring and storing the raw material in another buffer until the completion of the maintenance. If at a time epoch the production unit is found to be at the working condition $i, 0 \leq i \leq m$ and no preventive maintenance is initiated, an operating cost is incurred until the next time epoch which is equal to $c_{i}$, if the buffer is not empty, or to $\widetilde{c}_{i}$, if the buffer is empty. When a preventive or a corrective repair is performed, a repair cost is incurred, which is equal for each unit of time to $c_{p}$ or to $c_{f}$, respectively. We also assume that the cost of holding a unit of raw material in the buffer for one unit of time is equal to $h>0$. We consider that, if the buffer content is less than $K$ units, when a preventive or a corrective maintenance is completed, the production unit does not resume its operation immediately but it remains idle until the buffer is filled up. As soon as the buffer is filled up, the production unit resumes its operation normally by pulling $d$ units of the raw material from the buffer per unit of time. There are three possible actions $a \in\{0,1,2\}$. The action $a=0$ (allow the production unit to operate), the action $a=1$ (initiate a preventive maintenance of the production unit) and the action $a=2$ (initiate a corrective maintenance of the production unit). Let $m_{P M}$ and $m_{C M}$ be the expected times required for a preventive and a corrective maintenance, respectively. For the deteriorating production unit, we impose the same plausible conditions on the cost structure and on the transition probabilities as the one imposed for the deteriorating installation in the previous section for Model 1. For this problem, the state space of the system is the set $S=\{0,1, \ldots, m+1\} \times\{0, \ldots, K\}$, where $(i, x) \in S$ is the state in which $i$ is the working condition of the production unit and $x$ is the content of the buffer. We again consider a semi-Markov decision process in which we aim to find a policy that minimizes the long-run expected average cost per unit time. The decision epochs in this problem are the time epochs at which the system enters a state in $S$. Let $p_{s l}(a), a \in\{0,1,2\}$, be the probability that the state of the system at the next decision epoch will be the state $l \in S$, if the present state is $s \in S$ and the action $a \in\{0,1,2\}$ is selected and let $T(s, a)$ and $C(s, a)$ be the corresponding expected transition time and cost, respectively. These quantities are given below.

$$
\begin{aligned}
& p_{(i, x)(j, \max (x+p-d, 0))}(0)=p_{i j}, 0 \leq i \leq m, 0 \leq j \leq m+1,0 \leq x \leq K, \\
& T((i, x), 0)=1,0 \leq i \leq m, 0 \leq x \leq K,
\end{aligned}
$$


$C((i, x), 0)=c_{i}+h x+\frac{[d-\min (d, x+p)] C}{d}, 0 \leq i \leq m, 0<x \leq K$,

$C((i, 0), 0)=\widetilde{c}_{i}+\frac{(d-p) C}{d}, 0 \leq i \leq m$,

$p_{(i, x)(0, K)}(1)=1,0 \leq i \leq m, 0 \leq x \leq K$,

$T((i, x), 1)=m_{P M}+\int_{0}^{(K-x) / p}\left(\frac{K-x}{p}-t\right) f_{1}(t) d t$,

$0 \leq i \leq m, 0 \leq x \leq K$,

$C((i, x), 1)=c_{p} m_{P M}+C T((i, x), 1)+P \int_{(K-x) / p}^{\infty}(x+t p-K) f_{1}(t) d t$

$+\frac{h\left(K^{2}-x^{2}\right)}{2 p}+h K \int_{(K-x) / p}^{\infty}\left(t-\frac{K-x}{p}\right) f_{1}(t) d t$,

$0 \leq i \leq m, 0 \leq x \leq K$,

$p_{(m+1, x)(0, K)}(2)=1, \quad 0 \leq x \leq K$,

$T((m+1, x), 2)=m_{C M}+\int_{0}^{(K-x) / p}\left(\frac{K-x}{p}-t\right) f_{2}(t) d t$,

$0 \leq x \leq K$,

$C((m+1, x), 2)=c_{f} m_{C M}+C T((m+1, x), 2)$

$+P \int_{(K-x) / p}^{\infty}(x+t p-K) f_{2}(t) d t$

$+\frac{h\left(K^{2}-x^{2}\right)}{2 p}+h K \int_{(K-x) / p}^{\infty}\left(t-\frac{K-x}{p}\right) f_{2}(t) d t$,

$0 \leq x \leq K$.

We will explain in detail how (2) is derived. Suppose that the state of the system at a decision epoch is the state $(i, x), 0 \leq i \leq m, 0 \leq x \leq K$. If the action $a=1$ of preventive maintenance is initiated, then the state of the system at the next decision epoch will be the state $(0, K)$. The corresponding onestep expected cost $C((i, x), 1)$ consists of the expected maintenance cost, the expected lost production cost, the expected penalty cost and the expected holding cost. The expected costs due to the preventive maintenance and due to the lost production until the system reaches the state $(0, K)$ are equal to $c_{p} m_{P M}$ and to $C T((i, x), 1)$, respectively. By conditioning on the number of units of time that the preventive maintenance lasts, we deduce that the expected penalty cost and the expected holding cost, until the process reaches the state $(0, K)$, are equal to $P \int_{(K-x) / p}^{\infty}(x+t p-K) f_{1}(t) d t$ and $h \int_{0}^{(K-x) / p}(x+t p) d t+h K \int_{(K-x) / p}^{\infty}\left(t-\frac{K-x}{p}\right) f_{1}(t) d t$, respectively.
By summing the above four expressions, we obtain (2). A great number of numerical examples, in which the optimal policies were found by implementing the standard value-iteration algorithm for semi-Markov decision processes, provide strong evidence that, for each fixed buffer content $x \in\{0, \ldots, K\}$, the optimal policy is again of control-limit type. As for Model 1, in previous section, it seems again difficult to prove this conjecture.

\section{COMPUTATION OF THE AVERAGE COST UNDER A CONTROL-LIMIT POLICY}

Suppose that the installation in Model 1 and the production unit in Model 2 cannot be improved on their own, i.e. $p_{i j}=0$ if $i \in\{1, \ldots, m\}$ and $j<i$. In this case, the average cost of a control-limit policy (i.e. a policy that, for each fixed buffer level $x \in\{0, \ldots, K\}$, initiates a preventive maintenance of the installation in Model 1 or a preventive maintenance of the production unit in Model 2 if and only if the working condition of the installation in Model 1 or the working condition of the production unit in Model 2 is greater than or equal to a critical level $i(x))$ can be computed exactly using the usual regenerative argument (see, for example, Proposition 5.9 in Ross [15]). The exact computations of the average costs are achieved, since, for both models, the processes under a controllimit policy, are regenerative processes, where the successive entries into the state $(0,0)$ in Model 1 and into the state $(0, K)$ in Model 2, can be taken as regenerative epochs between successive cycles. Detailed descriptions of these exact computations are given in Section 4 of Karamatsoukis and Kyriakidis [9] paper for Model 1 and in Section 7 of Pavitsos and Kyriakidis [13] paper for Model 2. For both models, the average cost of a control-limit policy can be computed in the same way as in the case in which the preventive and corrective repair times are geometrically distributed with the following differences: (i) The expected times until the system, under a control-limit policy, enters the state $(0,0)$ in Model 1 or the state $(0, K)$ in Model 2, if the initial state is the state $(i, x), i(x) \leq i<m+1,0 \leq x \leq K$, are now equal to $T((i, x), 1)$. (ii) The expected times until the system, under a control-limit policy, enters the state $(0,0)$ in Model 1 or the state $(0, K)$ in Model 2, if the initial state is the state $(m+1, x), 0 \leq x \leq K$, are now equal to $T((m+1, x), 2)$. (iii) The expected costs until the system, under a control-limit policy, enters the state $(0,0)$ in Model 1 or the state $(0, K)$ in Model 2 , if the initial state is the state $(i, x), i(x) \leq i<m+1,0 \leq x \leq K$, are now equal to $C((i, x), 1)$. (iv) The expected costs until the system, under a control-limit policy, enters the state $(0,0)$ in Model 1 or the state $(0, K)$ in Model 2, if the initial state is the state $(m+1, x), 0 \leq x \leq K$, are now equal to $C((m+1, x), 2)$.

\section{NUMERICAL RESULTS}

In the following numerical examples, we implemented the standard value-iteration algorithms and the algorithms for the exact computation of the minimum average cost by running 
the corresponding Matlab programs on a personal computer equipped with an Intel Core $2 \mathrm{Duo}, 2.5 \mathrm{GHz}$ processor and 4 GB of RAM. For Model 1, in Examples 1 and 2, we assume that the repair times of the installation follow the Exponential, and the Weibull distribution, respectively. For Model 2, in Examples 3 and 4, we assume that the repair times of the production unit follow the Gamma and the Log-Normal distribution, respectively.

\section{Example 1 (Model 1).}

Suppose that $m=20, K=10, p=5, d=3, h=0.3, c_{f}=1.5$, $c_{p}=1, \quad c_{i}=0.1(i+1), \quad \tilde{c}_{i}=0.05(i+1), 0 \leq i \leq m$. We assume that the nonzero transition probabilities $p_{i j}, 0 \leq i, j \leq m+1$ are given by $p_{i j}=(m+2-i)^{-1}, i \leq j \leq m+1$. This means that if the present state of the installation is $i$, then the next state is uniformly distributed in the set $\{i, i+1, \ldots, m+1\}$. These probabilities satisfy Condition 5 since, for each $k=0, \ldots, m+1$, the quantity $\sum_{j=k}^{m+1} p_{i j}=\frac{m+2-k}{m+2-i}$ is increasing in $i, 0 \leq i \leq m$. We assume that the preventive and corrective repair times of the installation are exponentially distributed with parameters $\lambda_{1}>0$ and $\lambda_{2}>0$, respectively. Their probability density functions are given by

$$
f_{1}(t)=\lambda_{1} \exp \left(-\lambda_{1} t\right) \text { and } f_{2}(t)=\lambda_{2} \exp \left(-\lambda_{2} t\right), t \geq 0,
$$

respectively. We choose $\lambda_{1}=0.5$ and $\lambda_{2}=0.125$. Note that Condition 3 is satisfied since the mean of the Exponential distribution with parameter $\lambda>0$ is equal to $\lambda^{-1}$. The optimal policy obtained by the standard value-iteration algorithm is of control-limit type. The critical numbers $i^{*}(x), x \in\{0, \ldots, 10\}$, that characterize the optimal policy are given in Table 1 below.

Table 1. The critical numbers of the optimal policy

\begin{tabular}{c|ccccccccccc}
\hline$x$ & 0 & 1 & 2 & 3 & 4 & 5 & 6 & 7 & 8 & 9 & 10 \\
\hline$i^{*}(x)$ & 16 & 14 & 12 & 10 & 7 & 3 & 0 & 0 & 0 & 0 & 0 \\
\hline
\end{tabular}

The average cost of the optimal policy is found to be 2.1456 . We select as the prespecified accuracy number $\varepsilon$ for the stopping criterion of the value-iteration algorithm the value $\varepsilon=10^{-4}$. The algorithm is stopped after 65 iterations. The required computational time for the termination of the valueiteration algorithm was equal to 1.7472 seconds. The expected time and the expected cost of a cycle under this policy are equal to 4.3637 and 9.3628 , respectively.

\section{Example 2 (Model 1).}

Suppose that $m=15, K=8, p=11, d=10, c_{f}=2.5$ and $h=0.4$. We also assume that the operating costs $c_{i}, \widetilde{c}_{i}, 0 \leq i \leq m$ and the nonzero transition probabilities $p_{i j}, 0 \leq i, j \leq m+1$, are the same as in Example 1. We assume that the preventive and the corrective repair times of the installation follow the Weibull distribution with parameters $\alpha_{1}, \lambda_{1}>0$ and $\alpha_{2}, \lambda_{2}>0$, respectively. Their probability density functions are given by

$$
\begin{gathered}
f_{1}(t)=\alpha_{1} \lambda_{1}\left(\lambda_{1} t\right)^{\alpha_{1}-1} \exp \left[\left(-\lambda_{1} t\right)^{\alpha_{1}}\right] \text { and } \\
f_{2}(t)=\alpha_{2} \lambda_{2}\left(\lambda_{2} t\right)^{\alpha_{2}-1} \exp \left[\left(-\lambda_{2} t\right)^{\alpha_{2}}\right], t \geq 0
\end{gathered}
$$

respectively. We assume that $\alpha_{1}=1, \lambda_{1}=3$ and $\alpha_{2}=0.5, \lambda_{2}=5$. Note that Condition 3 is satisfied since the mean of the Weibull distribution with parameters $\alpha>0$ and $\lambda>0 \quad$ is $\quad \lambda^{-1} \Gamma\left(1+\alpha^{-1}\right)$, where $\Gamma(x)=\int_{0}^{\infty} t^{x-1} \exp (-t) d t$, $x>0$, is the Gamma function. In Table 2 below, we present the critical numbers of the optimal control-limit policy obtained by the value-iteration algorithm for the values of $c_{p}$ in the set $\{1.2,1.5,1.8,2,2.3,2.5\}$.

Table 2. The form of the optimal policy as $c_{p}$ varies $c_{p}=1.2$

\begin{tabular}{l|ccccccccc}
\hline$x$ & 0 & 1 & 2 & 3 & 4 & 5 & 6 & 7 & 8 \\
\hline$i^{*}(x)$ & 16 & 14 & 10 & 6 & 1 & 0 & 0 & 0 & 0 \\
\hline \multicolumn{10}{c}{$c_{p}=1.5$} \\
\hline
\end{tabular}

\begin{tabular}{l|ccccccccc}
\hline$x$ & 0 & 1 & 2 & 3 & 4 & 5 & 6 & 7 & 8 \\
\hline$i^{*}(x)$ & 16 & 14 & 11 & 6 & 1 & 0 & 0 & 0 & 0 \\
\hline
\end{tabular}
$c_{p}=1.8$

\begin{tabular}{l|ccccccccc}
\hline$x$ & 0 & 1 & 2 & 3 & 4 & 5 & 6 & 7 & 8 \\
\hline$i^{*}(x)$ & 16 & 14 & 11 & 6 & 2 & 0 & 0 & 0 & 0 \\
\hline \multicolumn{1}{c}{$c_{p}=2$} \\
\hline$x$ & 0 & 1 & 2 & 3 & 4 & 5 & 6 & 7 & 8 \\
\hline$i^{*}(x)$ & 16 & 15 & 11 & 7 & 2 & 0 & 0 & 0 & 0 \\
\hline & \multicolumn{10}{c}{$c_{p}=2.3$} \\
\hline
\end{tabular}

\begin{tabular}{l|ccccccccc}
\hline$x$ & 0 & 1 & 2 & 3 & 4 & 5 & 6 & 7 & 8 \\
\hline$i^{*}(x)$ & 16 & 15 & 11 & 7 & 2 & 0 & 0 & 0 & 0 \\
\hline \multicolumn{8}{c}{$c_{p}=2.5$} \\
\hline$x$ & 0 & 1 & 2 & 3 & 4 & 5 & 6 & 7 & 8 \\
\hline$i^{*}(x)$ & 16 & 15 & 12 & 7 & 2 & 0 & 0 & 0 & 0 \\
\hline
\end{tabular}

From Table 2, it can be seen that for each fixed buffer level $x$, the critical number $i^{*}(x)$ remains unchanged or increases as $c_{p}$ increases. In Table 3 , for the above values of $c_{p}$, we present the average cost of the optimal policy obtained by the 
value-iteration algorithm. We also present the expected time and the expected cost of a cycle under the optimal policy for the exact computation of its average cost.

Table 3. The effect of varying $c_{p}$

\begin{tabular}{|c|c|c|c|}
\hline$c_{p}$ & Average Cost & $\begin{array}{c}\text { Expected } \\
\text { Time }\end{array}$ & $\begin{array}{c}\text { Expected } \\
\text { Cost }\end{array}$ \\
\hline 1.2 & 1.6293 & 2.4869 & 4.0519 \\
\hline 1.5 & 1.6623 & 2.5493 & 4.2376 \\
\hline 1.8 & 1.6942 & 2.5493 & 4.3190 \\
\hline 2 & 1.7146 & 2.6219 & 4.4955 \\
\hline 2.3 & 1.7449 & 2.6219 & 4.5749 \\
\hline 2.5 & 1.7642 & 2.6949 & 4.7545 \\
\hline
\end{tabular}

From Table 3, it can be seen, as expected, that the average cost of the optimal policy and also, the expected time and the expected cost of a cycle under the optimal policy, either increase or remain unchanged, as $c_{p}$ increases.

\section{Example 3 (Model 2).}

Suppose that $m=15, p=5, d=8, C=10, c_{f}=30, c_{p}=20$, $c_{i}=6(i+1), \widetilde{c}_{i}=3(i+1), 0 \leq i \leq m$. The nonzero transition probabilities $p_{i j}, 0 \leq i, j \leq m+1$, are the same as in Examples 1 and 2. We assume that the preventive and the corrective repair times of the production unit follow the Gamma distribution with parameters $\alpha_{1}, \lambda_{1}>0$ and $\alpha_{2}, \lambda_{2}>0$, respectively. Their probability density functions are given by

$$
\begin{gathered}
f_{1}(t)=\left[\Gamma\left(\alpha_{1}\right)\right]^{-1} \lambda_{1}^{\alpha_{1}} t^{\alpha_{1}-1} \exp \left(-\lambda_{1} t\right) \text { and } \\
f_{2}(t)=\left[\Gamma\left(\alpha_{2}\right)\right]^{-1} \lambda_{2}^{\alpha_{2}} t^{\alpha_{2}-1} \exp \left(-\lambda_{2} t\right), t \geq 0,
\end{gathered}
$$

respectively, where, $\Gamma(\alpha)$ is the Gamma function. We assume that $\alpha_{1}=4, \lambda_{1}=2$ and $\alpha_{2}=14, \lambda_{2}=2$. Note that Condition 3 is satisfied since the mean of the Gamma distribution with parameters $\alpha>0$ and $\lambda>0$ is $\alpha \lambda^{-1}$. It is interesting to examine the effect of the variation of $K$ on the average cost of the optimal policy. If the value of $K$ is small, it is probable that the buffer is filled up quickly during a maintenance causing a high penalty cost. If the value of $K$ is large, a high storage cost is incurred. In Table 4 below, we present, for different values of $K$, the average cost $g(K)$ of the optimal policy for the following four cases: (i) $h=3, P=15$, (ii) $h=3, P=0$, (iii) $h=0, P=15$ and (iv) $h=0, P=0$.

Table 4. The minimum average cost $g(K)$ as $K$ varies

\begin{tabular}{|c|c|c|c|c|}
\hline$K$ & $h=3, P=15$ & $h=3, P=0$ & $h=0, P=15$ & $h=0, P=0$ \\
\hline 1 & 68.9558 & 26.8800 & 66.6215 & 24.1981 \\
\hline 3 & 66.0687 & 30.9942 & 59.1483 & 23.4994 \\
\hline 5 & 69.1509 & 36.9032 & 57.1031 & 23.6317 \\
\hline 7 & 72.5769 & 42.5994 & 55.2288 & 23.5936 \\
\hline 9 & 76.7519 & 48.2473 & 54.3736 & 23.5765 \\
\hline
\end{tabular}

\begin{tabular}{|l|c|c|c|c|}
\hline 11 & 82.5085 & 54.2463 & 54.3242 & 23.5777 \\
\hline 13 & 88.1169 & 60.1858 & 54.2316 & 23.5762 \\
\hline 15 & 93.8200 & 66.1003 & 54.2069 & 23.5769 \\
\hline 17 & 99.7849 & 72.1025 & 54.2063 & 23.5769 \\
\hline 19 & 105.7056 & 78.0866 & 54.2036 & 23.5769 \\
\hline 21 & 111.6292 & 84.0622 & 54.2029 & 23.5769 \\
\hline 23 & 117.6178 & 90.0627 & 54.2026 & 23.5769 \\
\hline 25 & 123.5942 & 96.0572 & 54.2023 & 23.5769 \\
\hline
\end{tabular}

From Table 4, it can be seen that, in the first case the minimum average cost decreases for $1 \leq K \leq 3$ and increases for $K \geq 3$. The minimum average cost is achieved at $K=3$ and it is equal to 66.0687. In Case (ii), $g(K)$ is increasing with respect to $K$, in Case (iii), $g(K)$ is decreasing with respect to $K$ and in Case (iv), $g(K)$ is constant for $K \geq 15$. The graphs of $g(K)$ as a function of $K$ for the four cases are given in Figure 2.

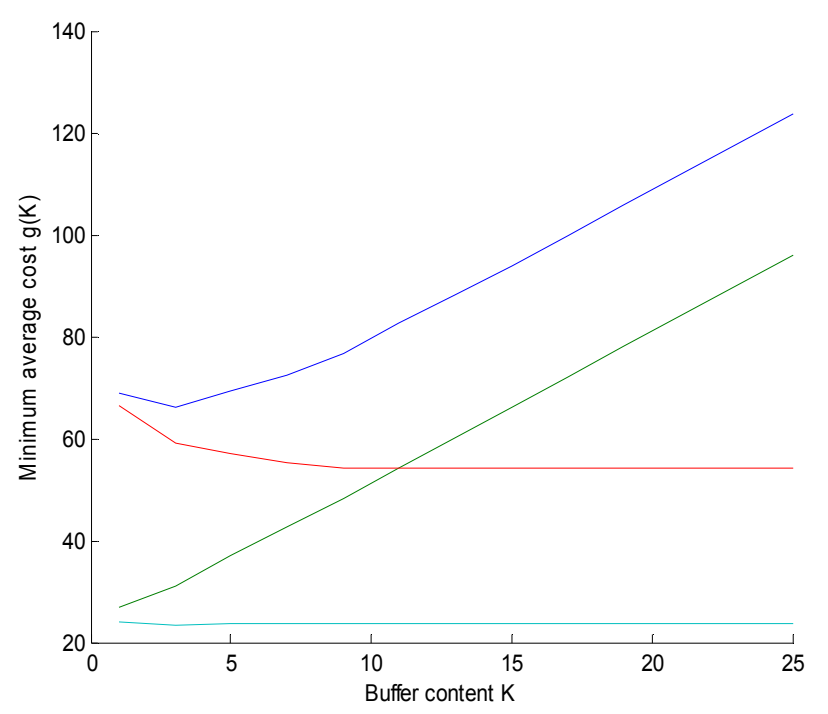

Fig. 2. The graph of $g(K)$

The blue line corresponds to Case (i), the green line corresponds to Case (ii), the red line corresponds to Case (iii) and the cyan line corresponds to Case (iv).

\section{Example 4 (Model 2).}

Suppose that $m=25, K=10, d=8, C=10, c_{f}=16, c_{p}=9$, $h=4, P=12$. We also assume that the operating costs $c_{i}, \widetilde{c}_{i}, 0 \leq i \leq m$ and the nonzero transition probabilities $p_{i j}, 0 \leq i, j \leq m+1$, are the same as in Example 3. We consider that the preventive and the corrective repair times of the production unit follow the Log-Normal distribution with parameters $\mu_{1} \in \mathfrak{R}, \sigma_{1}^{2}>0$ and $\mu_{2} \in \mathfrak{R}, \sigma_{2}^{2}>0$, respectively. Their probability density functions are given by 


$$
\begin{gathered}
f_{1}(t)=\left[t \sigma_{1} \sqrt{2 \pi}\right]^{-1} \exp \left\{-\frac{\left(\ln (t)-\mu_{1}\right)^{2}}{2 \sigma_{1}^{2}}\right\} \text { and } \\
f_{2}(t)=\left[t \sigma_{2} \sqrt{2 \pi}\right]^{-1} \exp \left\{-\frac{\left(\ln (t)-\mu_{2}\right)^{2}}{2 \sigma_{2}^{2}}\right\}, t>0,
\end{gathered}
$$

respectively. We assume that $\mu_{1}=9, \sigma_{1}=5$ and $\mu_{2}=12, \sigma_{2}=6$. Note that Condition 3 is satisfied since the mean of the Log-Normal distribution with parameters $\mu \in \mathfrak{R}$ and $\sigma^{2}>0$ is equal to $\exp \left(\mu+\frac{1}{2} \sigma^{2}\right)$. It seems interesting to examine the effect of the variation of the replenishment rate $p$ on the form of the optimal policy and on the minimum average cost $g(p)$. In Table 5, we present, for various values of $p$, the critical numbers $i^{*}(x), 0 \leq x \leq 10$, that characterize the optimal policy and the corresponding minimum average cost $g(p)$.

Table 5. The optimal policy and its average cost as $p$ varies

\begin{tabular}{|c|l|l|}
\hline$p$ & $\begin{array}{c}\text { Average } \\
\text { Cost } g(p)\end{array}$ & $i^{*}(x), 0 \leq x \leq 10$ \\
\hline 1 & 74.4762 & $\begin{array}{l}101116212325 \\
2626252422\end{array}$ \\
\hline 2 & 84.5467 & $\begin{array}{l}151216192123 \\
2423222019\end{array}$ \\
\hline 3 & 95.3609 & $\begin{array}{l}181416182122 \\
2120191817\end{array}$ \\
\hline 4 & 109.3707 & 211618202120 \\
& & 2319181716 \\
\hline 5 & 122.2072 & 1918171615 \\
\hline 6 & 136.2852 & 242122202019 \\
& & 2517161615 \\
\hline 7 & 148.5111 & 1717161615 \\
\hline
\end{tabular}

If $i^{*}(x)=m+1$, for some buffer level $x \in\{0, \ldots, K\}$, then a preventive maintenance is never initiated whenever the buffer content is equal to $x$. From Table 5, we observe that the minimum average cost increases, as $p$ increases. We also observe that, for constant buffer level $x$, the critical number $i^{*}(x)$ is not a monotone function with respect to $p$.

\section{REFERENCES}

[1] K.J. Bassey and P.E. Chigbu, "On optimal control theory in marine oil spill management: A Markovian decision approach,” Eur. J. Oper. Res., Vol. 217, Iss. 2, pp. 470-478, 2012.

[2] Z. Benyamini and U. Yechiali, "Optimality of control limit maintenance policies under non-stationary deterioration," Probab. Eng. Inform. Sc., Vol. 13, pp. 55-70, 1999.

[3] J.S. Borrero and R. Akhavan-Tabatabaei, "Time and inventory dependent optimal maintenance policies for single machine workstations: An MDP approach,” Eur. J. Oper. Res., Vol. 228, Iss. 3, pp. 545-555, 2013.

[4] M. Chen and R.M. Feldman, "Optimal replacement policies with minimal repair and age-dependent costs," Eur. J. Oper. Res., Vol. 98, Iss. 1, pp. 75-84, 1997.

[5] N. Douer and U. Yechiali, "Optimal repair and replacement in Markovian systems," Stoch. Models, Vol. 10, pp. 253-270, 1994.

[6] A. Federgruen and K.S. So, "Optimal time to repair a broken server," Adv. Appl. Probab., Vol. 21, pp. 376-397, 1989.

[7] A. Grosfeld-Nir, "Control limits for two-state partially observable Markov decision processes,” Eur. J. Oper. Res., Vol. 182, pp. 300-304, 2007.

[8] Y. Huang and X. Guo, "Finite horizon semi-Markov decision processes with application to maintenance systems," Eur. J. Oper. Res., Vol. 212, Iss. 1, pp. 131-140, 2012.

[9] C.C. Karamatsoukis and E.G. Kyriakidis, "Optimal maintenance of a production-inventory system with idle periods," Eur. J. Oper. Res., Vol. 96, pp. 744-751, 2009.

[10] M. Kurt and J.P. Kharoufeh, "Monotone optimal replacement policies for a Markovian deteriorating system in a controllable environment," Oper. Res. Lett., Vol. 38, Iss. 4, pp. 273-279, 2010.

[11] M. Kurt and J.P. Kharoufeh, "Optimally maintaining a Markovian deteriorating system with limited imperfect repairs," Eur. J. Oper. Res., Vol. 205, Iss. 2, pp. 368-380, 2010.

[12] M.S. Moustafa, E.Y. Maksoud Abdel and S. Sadek, "Optimal major and minimal maintenance policies for deteriorating systems," Reliab. Eng. Syst. Safe., Vol. 83, pp. 363-368, 2004.

[13] A. Pavitsos and E.G. Kyriakidis, "Markov decision models for the optimal maintenance of a production unit with an upstream buffer," Comput. Oper. Res., Vol. 36, pp. 1993-2006, 2009.

[14] A.B. Piunovskiy, "Controlled Stochastic Jump Processes," WSEAS conferences, Tenerife, Math. Mod. Eng. Sci. MMES-16, pp. 104-112, 2010.

[15] S.M. Ross, Applied Probability Models with Optimization Applications. Dover, New York, 1992.

[16] H.C. Tijms, Stochastic Models: An Algorithmic Approach. Wiley, Chichester, 1994.

[17] C.L. Tomasevicz and S. Asgarpoor, "Optimum maintenance policy using semi-Markov decision processes," Electr. Pow. Syst. Res., Vol. 79, Iss. 9, pp. 1286-1291, 2009.

[18] F.A. Van Der Duyn Schouten and S.G. Vanneste, "Maintenance optimization of a production system with buffer capacity," Eur. J. Oper. Res., Vol. 82, pp. 419-446, 1995.

[19] H. Wang, "A survey of maintenance policies of deteriorating systems," Eur. J. Oper. Res., Vol. 139, pp. 469-489, 2002.

[20] X. Zhang and H. Gao, "Road maintenance optimization through a discrete-time semi-Markov decision process," Reliab. Eng. Syst. Safe., Vol. 103, pp. 110-119, 2012.

\section{Creative Commons Attribution License 4.0 (Attribution 4.0 International, CC BY 4.0)}

This article is published under the terms of the Creative Commons Attribution License 4.0 https://creativecommons.org/licenses/by/4.0/deed.en US 